\title{
A robust post-processing method to determine skin friction in turbulent boundary layers from the velocity profile
}

\author{
Eduardo Rodríguez-López ${ }^{1}$ Paul J. K. Bruce ${ }^{1}$ Oliver R. H. Buxton ${ }^{1}$
}

Received: 1 December 2014 / Revised: 30 January 2015 / Accepted: 25 February 2015 / Published online: 17 March 2015

(C) The Author(s) 2015. This article is published with open access at Springerlink.com

\begin{abstract}
The present paper describes a method to extrapolate the mean wall shear stress, $\tau_{\text {wall }}$, and the accurate relative position of a velocity probe with respect to the wall, $\Delta y$, from an experimentally measured mean velocity profile in a turbulent boundary layer. Validation is made between experimental and direct numerical simulation data of turbulent boundary layer flows with independent measurement of the shear stress. The set of parameters which minimize the residual error with respect to the canonical description of the boundary layer profile is taken as the solution. Several methods are compared, testing different descriptions of the canonical mean velocity profile (with and without overshoot over the logarithmic law) and different definitions of the residual function of the optimization. The von Kármán constant is used as a parameter of the fitting process in order to avoid any hypothesis regarding its value that may be affected by different initial or boundary conditions of the flow. Results show that the best method provides an accuracy of $\Delta u_{\tau} \leq 0.6 \%$ for the estimation of the friction velocity and $\Delta y^{+} \leq 0.3$ for the position of the wall. The robustness of the method is tested including unconverged near-wall measurements, pressure gradient, and reduced number of points; the importance of the location of the first point is also tested, and it is shown that the method presents
\end{abstract}

Eduardo Rodríguez-López

eduardo.rodriguez-lopez12@imperial.ac.uk

Paul J. K. Bruce

p.bruce@imperial.ac.uk

Oliver R. H. Buxton

o.buxton@imperial.ac.uk

1 Department of Aeronautics, Imperial College London, SW7 2AZ London, UK a high robustness even in highly distorted flows, keeping the aforementioned accuracies if one acquires at least one data point in $y^{+}<10$. The wake component and the thickness of the boundary layer are also simultaneously extrapolated from the mean velocity profile. This results in the first study, to the knowledge of the authors, where a five-parameter fitting is carried out without any assumption on the von Kármán constant and the limits of the logarithmic layer further from its existence.

\section{Introduction}

Dimensional analysis applied to wall-bounded flows shows that the mean velocity profile, or equivalently the velocity gradient, can be described by just two non-dimensional parameters. Without any assumption, we can write that $\frac{\mathrm{d} u}{\mathrm{~d} y}=\frac{u_{\tau}}{y} \Phi\left(\frac{y}{\delta_{v}}, \frac{y}{\delta}\right)$, where $u$ is the mean velocity (throughout the whole paper, lower case letters will refer to mean quantities), $\delta_{v}=v / u_{\tau}$ is the viscous lengthscale, $u_{\tau}=\sqrt{\tau_{\text {wall }} / \rho}$ is the friction velocity, and $\delta$ is the thickness of the boundary layer or the half-width of the channel (Schlichting and Gersten 2000; Pope 2000). As Prandtl first postulated, at sufficiently high Reynolds number, there is an inner layer in which the velocity profile is described by the viscous scales independently of $\delta$ and $u_{e}$, the freestream velocity; $\frac{\mathrm{d} u^{+}}{\mathrm{d} y^{+}}=\Phi_{\text {inner }}\left(y^{+}\right)$, where $y^{+}=y / \delta_{v}$ and $u^{+}=u / u_{\tau}$. Similarly, for the outer layer, the velocity profile is independent of $v$ implying that $\frac{\mathrm{d} u^{+}}{\mathrm{d} y^{+}}=\Phi_{\text {outer }}(y / \delta)$. One can argue easily that for $\delta_{v} \ll y \ll \delta$ : $\Phi_{\text {inner }}\left(y^{+}\right)=\Phi_{\text {outer }}(y / \delta)$. This condition can only be satisfied by both functions being a constant (Millikan 1938), thus resulting in $\frac{\mathrm{d} u^{+}}{\mathrm{d} y^{+}}=\frac{1}{\kappa y^{+}}$, where $\kappa$ is the von Kármán constant. It can thus be integrated to obtain the logarithmic law for the velocity $u^{+}=\frac{1}{\kappa} \ln \left(y^{+}\right)+B$, where $B$ is an integration constant. 
As seen above, the most significant velocity scale of the velocity profile in the turbulent boundary layer is $u_{\tau}$. From an experimental point of view, the direct determination of the friction velocity presents a high degree of difficulty (Klewicki 2007; Hutchins and Choi 2002); therefore, the possibility to extrapolate this magnitude from the velocity profile is of particular relevance. With respect to the wall normal distance, the scaling parameter is $\delta_{v}=v / u_{\tau}$, which usually is of the same order of magnitude as the typical uncertainties that can be found on the wall normal position determination of a typical velocity probe. Orlu et al. (2010) or Salari and Tabar (2011), for instance, provide reviews of the common experimental techniques to accurately measure the wall location. The accuracies reported by different authors present a high dispersion with errors spanning from $\pm 5 \mu \mathrm{m}$ (Tay et al. 2012) to $\pm 250 \mu \mathrm{m}$ (Ryan et al. 2011), corresponding to approximately $\pm 0.2 \delta_{v}$ to $\pm 4 \delta_{v}$, respectively. Additionally, these methodologies are usually applied under zero-velocity conditions; further deformations of the prong or of the traverse system due to the flow introduce further uncertainty on the measurement procedure.

From the dimensional analysis presented, it can be seen that the inner region, dominated by the viscosity, scales as $\delta_{v}$ and $u_{\tau}$. In particular, the velocity profile behaves as $u^{+}=y^{+}$, and broad experimental evidence (Schlichting and Gersten 2000; Pope 2000; Hutchins and Choi 2002) shows that this expression is valid until $y^{+} \simeq 5$. This result has been used by several authors to extrapolate the friction velocity and the wall normal location (Hutchins and Choi 2002; Orlu et al. 2010; Tay et al. 2012). However, this approach requires that special care is taken to account for errors due to the wall effect when using hot wires (Chew et al. 1998; Hutchins and Choi 2002), limiting the range of validity to $3.5<y^{+}<5$. Alfredsson et al. (2011) use the similarity of the cumulative probability density function in the near-wall region to extrapolate $u_{\tau}$ and $\Delta y$. The accuracy of all of these methods to determine $\tau_{\text {wall }}$ and $\Delta y$ decreases drastically for increasing distance of the first measured point with respect to the wall.

Although the published data seem to point toward the commonly accepted description of $u^{+}=y^{+}$for the inner layer (Schlichting and Gersten 2000; Pope 2000; Marusic et al. 2010), the outer part of wall-bounded flows (scaling on the thickness $\delta$ ) is assumed to present a high dependence on boundary and initial conditions. Coles (1956) was the first author to consider the description of the outer part of the boundary layer as a wake function added to the description of the inner layer $\left(u^{+}=u_{\text {inner }}^{+}+\frac{\Pi}{\kappa} \mathcal{W}(y / \delta)\right)$. Several different mathematical formulations of the wake function, $\mathcal{W}$, can be found in the literature (Chauhan et al. 2007) resulting in different values of the wake component, П. These same authors (Chauhan et al. 2009) make use of the fact that the wake component is greatly affected by boundary or initial conditions, such as the pressure gradient in order to use $\Pi$ as a diagnostic quantity to assess the validity of experiments. The influence of the pressure gradient in the wake region is also studied by Nickels (2004) who proposes a different canonical description of the velocity profile. Mehdi et al. (2013) develop an integral method to estimate the friction velocity and the wall position showing that the role played by the outer part of the boundary layer in the determination of $u_{\tau}$ is secondary.

Following Millikan (1938) argument, the logarithmic layer, also called overlap region, is the part of the velocity profile, which adapts the two aforementioned scalings. From this point of view, $y^{+} \frac{\mathrm{d} u^{+}}{\mathrm{d} y^{+}}=\Phi_{\text {inner }}\left(y / \delta_{v}\right)=\Phi_{\text {outer }}(y / \delta)$; therefore, $\frac{\mathrm{d} u^{+}}{\mathrm{d} y^{+}}=\frac{1}{\kappa y^{+}}=$constant is the only restriction applying. It does not provide information regarding whether this constant should be the same independent of the type of the flow or the boundary conditions nor its limits. Moreover, if the logarithmic law adapts inner and outer regions of the boundary layer, regarding that the outer flow is affected by boundary conditions, one can expect that the logarithmic region is as well affected by those boundary conditions. It is possible that these changes can be reflected in the constants of the logarithmic law, namely the von Kármán constant, $\kappa$ and the integration constant $B$.

As possible examples of the values that the von Kármán constant has taken through the years in the literature, one can cite that von Kármán (1930) assigned the value of 0.38 to this constant. Some years after, Coles (1956) used $\kappa=0.40$ and assessed that in practically all cases where equation [log-law] is explicitly taken as a definition, $\kappa$ is found to lie between 0.39 and 0.41, Zagarola et al. (1997) assume $\kappa=0.436$, Österlund (1999) uses $\kappa=0.38$, Schlichting and Gersten (2000) give the value $\kappa=0.41$, whereas Pope (2000) gives a margin of $\pm 5 \%$ to that value, Zanoun et al. (2003) provide a summary of the values used from 1930 until 2000 comprising $0.33 \leq \kappa \leq 0.43$ and show that from their data, $\kappa=0.37(\sim 1 / e)$ independently of Reynolds number. McKeon et al. (2004) study pipe flow at very high Reynolds number and show $\kappa=0.421$. Some years after, Nagib and Chauhan (2008) included published data of the von Kármán constant spanning $0.3 \leq \kappa \leq 0.6$ and showed different asymptotic values for pipe flow and boundary layer flow $(\kappa=0.41$ and 0.384 , respectively) as well as a dependence on the pressure gradient. More recent publication by Marusic et al. (2013) presents $\kappa=0.39 \pm 0.02$ and shows that due to uncertainty in the measurements, its value cannot be discerned inside this range. Similar dispersion is obtained by Bailey et al. (2014) who shows $\kappa=0.40 \pm 0.02$ for pipe flow. Along this line, Segalini et al. (2013) demonstrate that the uncertainty on the determination of the von 
Kármán constant is, at least, the same of that in the friction velocity.

As one can see, the value (and the universality) of this constant has been subjected to question from the scientific community since its appearance. This debate is important not only from a fundamental point of view, but also from, as Orlu et al. (2010) show, the wall position and the shear stress, and other quantities which can be extrapolated from the mean velocity profile present a dependence on the chosen von Kármán constant. Therefore, the selection of one or other value appears as one of the sources of uncertainty in any method extrapolating magnitudes from the velocity profile. For instance, Klewicki (2007) assesses that the von Kármán constant [has to] be chosen judiciously when referring to the Clauser (1954) method.

Even though one can see that the constants of the logarithmic layer are subject to debate, several authors have used the description of the velocity profile on this layer in order to extrapolate the scaling magnitudes of the boundary layer. One can find as the first, and most broadly extended, method the Clauser plot (Clauser 1954), which provides an accuracy of $\pm 5 \%$ for the friction velocity, better for increasing Reynolds number (Klewicki 2007; Wei et al. 2005). Note that in any case, as Orlu et al. (2010) mention, the use of the Clauser plot cannot be seen as a measure of the wall stress but just as an estimation of it from the velocity profile. Also, since it does not take information of the inner part of the boundary layer, it does not allow the extrapolation of the wall location.

In order to do so, we have to consider a continuous description for the mean velocity profile including inner, buffer, and logarithmic layers. Nickels (2004), Orlu et al. (2010), or Chauhan et al. (2007) consider different strategies for this description concluding, as well as other authors (Chauhan et al. 2009; Nagib and Chauhan 2008; Monkewitz et al. 2007), that the Musker velocity profile provides the best solution to the problem (Musker 1979). Figure 1a shows the buffer layer adapting from the linear velocity profile to the logarithmic layer. Musker (1979) described this adaptation region as a continuous change in the eddy viscosity from the wall to the overlap region. Note that by definition $u_{\text {musker }}^{+} \leq u_{\text {log }}^{+}$since the solution is calculated to asymptotically match the logarithmic layer.

Kendall and Koochesfahani (2008) assess an accuracy of $\pm 1 \%$ in the friction velocity extrapolated from the velocity profile and mention that this method can be used to detect the wall location with an accuracy of $\pm 5 \mu \mathrm{m}$. However, most of the results presented in those papers are obtained for a fixed $\Delta y=0$, considering only $u_{\tau}$ as a fitting parameter. They also demonstrate that allowing the wall position to vary as an optimization parameter has an effect around $0.25 \%$ in the estimation of the friction velocity, but they do not assess the influence of the optimization of the wall stress on the accuracy of $\Delta y$. This dependency was later studied by Orlu et al. (2010) who reported errors of the order of $\Delta y \sim 0.5 \delta_{v}$ independently of considering or not $u_{\tau}$ as an optimization parameter. Table 1 provides a summary of the different quantities that various researchers have extrapolated from the mean velocity profile and the parts of the velocity profile that have been included for this extrapolation.

The difficulty of choosing the appropriate von Kármán constant for given boundary conditions introduces further uncertainty to the methods. The problem can be summarized as mentioned by Chauhan et al. (2007) in the abstract : even an accurate composite representation must

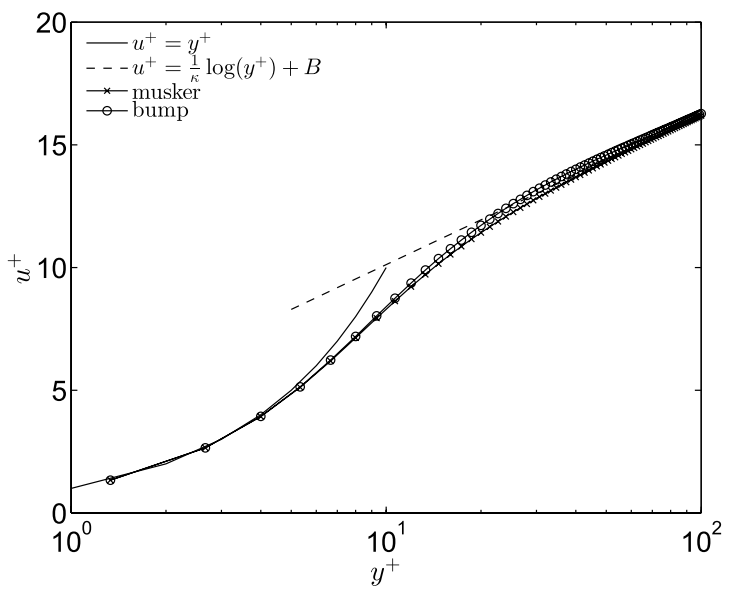

(a)

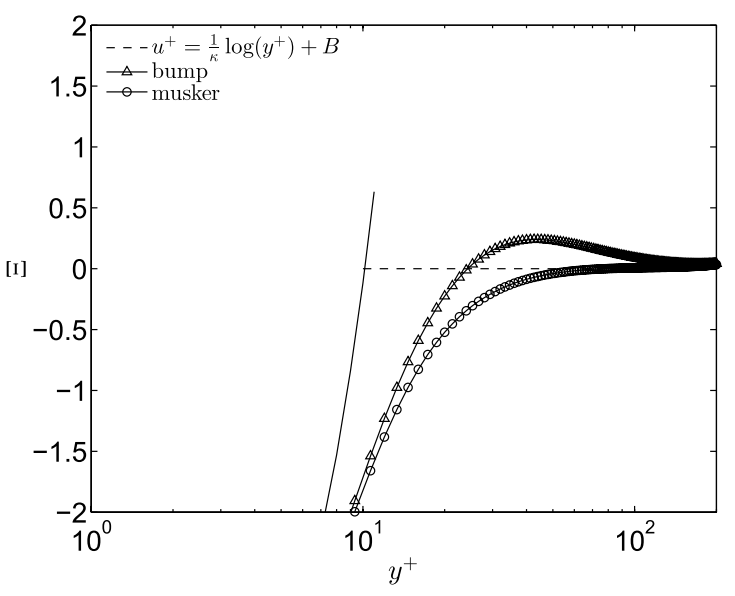

(b)

Fig. 1 Detail of mean velocity profile. a Detail on the buffer region for Musker $(\times)$ velocity profile, $\mathbf{b} \Xi=u^{+}-\left[\frac{1}{\kappa} \ln \left(y^{+}\right)+B\right]$ for $\operatorname{bump}(\triangle)$ and Musker (o) velocity profiles 
Table 1 Parameters extrapolated from the mean velocity profile and parts of the profile which have been considered for the optimization in the literature

\begin{tabular}{|c|c|c|c|c|c|c|c|c|c|}
\hline & \multicolumn{4}{|c|}{ Used part of velocity profile } & \multicolumn{5}{|c|}{ Parameters optimized } \\
\hline & Viscous & Buffer & $\log$ & Wake & $u_{\tau}$ & $\Delta y$ & $\Pi$ & $\delta$ & $\kappa$ \\
\hline Clauser (1954) & $x$ & $x$ & $\checkmark$ & $x$ & $\checkmark$ & $x$ & $x$ & $x$ & $x$ \\
\hline Coles (1956) & $x$ & $x$ & $\checkmark$ & $\checkmark$ & $x$ & $x$ & $\checkmark$ & $\checkmark$ & $x$ \\
\hline Coles (1968) & $x$ & $x$ & $\checkmark$ & $\checkmark$ & $\checkmark$ & $x$ & $\checkmark$ & $\checkmark$ & $x$ \\
\hline Durst et al. (1996) & $\checkmark$ & $\checkmark$ & $x$ & $x$ & $\checkmark$ & $\checkmark$ & $x$ & $x$ & $x$ \\
\hline Hutchins and Choi (2002) & $\checkmark$ & $x$ & $x$ & $x$ & $\checkmark$ & $\checkmark$ & $x$ & $x$ & $x$ \\
\hline Nickels (2004) & $\checkmark$ & $\checkmark$ & $\checkmark$ & $\checkmark$ & $x$ & $x$ & $\checkmark$ & $\checkmark$ & $\checkmark$ \\
\hline Kendall and Koochesfahani (2008) & $\checkmark$ & $\checkmark$ & $\checkmark$ & $x$ & $\checkmark$ & $\checkmark$ & $x$ & $\times$ & $x$ \\
\hline Nagib et al. (2007) & $\checkmark$ & $\checkmark$ & $\checkmark$ & $x$ & $\checkmark$ & $\times$ & $\times$ & $x$ & $x$ \\
\hline Chauhan et al. (2007) & $\checkmark$ & $\checkmark$ & $\checkmark$ & $\checkmark$ & $\checkmark$ & $x$ & $x$ & $x$ & $x$ \\
\hline Nagib and Chauhan (2008) & $\checkmark$ & $\checkmark$ & $\checkmark$ & $\checkmark$ & $x$ & $\times$ & $x$ & $\times$ & $\checkmark$ \\
\hline Chauhan et al. (2009) & $\checkmark$ & $\checkmark$ & $\checkmark$ & $\checkmark$ & $\checkmark$ & $\times$ & $\checkmark$ & $\checkmark$ & $x$ \\
\hline Orlu et al. (2010) & $\checkmark$ & $\checkmark$ & $\checkmark$ & $x$ & $\times$ & $\checkmark$ & $\times$ & $x$ & $\checkmark$ \\
\hline Tay et al. (2012) & $\checkmark$ & $x$ & $x$ & $x$ & $x$ & $\checkmark$ & $x$ & $x$ & $x$ \\
\hline Vinuesa (2013) & $\checkmark$ & $\checkmark$ & $\checkmark$ & $x$ & $x$ & $\checkmark$ & $x$ & $x$ & $\checkmark$ \\
\hline Present paper & $\checkmark$ & $\checkmark$ & $\checkmark$ & $\checkmark$ & $\checkmark$ & $\checkmark$ & $\checkmark$ & $\checkmark$ & $\checkmark$ \\
\hline
\end{tabular}

utilize the [...] accurate values of its parameters $\kappa$ and $B$ for the given flow geometry. The way to approach this obstacle in the present paper follows the argument shown above, specifically if we consider the logarithmic layer as the adaptation between the inner layer and the outer part. Since this outer region depends on the boundary conditions, it forces the overlap region to be dependent on those boundary conditions. The information of these conditions is already contained in the measured velocity profile; thus, the present paper will leave the von Kármán constant free to adopt the value that best suits the flow without further hypothesis than the existence of the overlap region between inner and outer scalings. This hypothesis is also supported by the studies of Nagib and Chauhan (2008) where $\kappa$ is shown to present a dependence on the type of flow and the pressure gradient. The information of the type of flow, pressure gradient, or any other source of change in the von Kármán constant is already included in the velocity profile; therefore, it seems reasonable leaving $\kappa$ as an optimization parameter rather than forcing it to be a somehow arbitrary value based on previous studies (which might not match the exact physics of that particular flow).

Summarizing the present paper presents a method to extrapolate the friction velocity $\left(u_{\tau}\right)$, a possible wall offset $(\Delta y)$, the von Kármán constant $(\kappa)$, the wake component $(\Pi)$, and the thickness of the boundary layer $(\delta)$ from one measured experimental velocity profile. A note of caution has to be made at this point. Most of the studies summarized in Table 1 did not perform an optimization on all five parameters simultaneously for a diverse range of reasons. Among them may be the fact that the skin friction was directly measured, e.g., Österlund (1999), or the studies were simply only concerned with outer variables, e.g., Nickels (2004), or inner variables, e.g., Hutchins and Choi (2002), only. The novelty of the present approach is that by optimizing for all five parameters $\left\{u_{\tau}, \Delta y, \kappa, \Pi, \delta\right\}$, no assumptions need to be made $a$ priori as to the nature of the velocity profile other than the existence of an logarithmic layer and the empirically observed relation of Eq. 3 (which will be discussed further in Sect. 2). In particular, no a priori knowledge of the extent of the logarithmic layer is required. This means that the method is exceptionally robust, not only for canonical turbulent boundary layers but also to low Reynolds number and nonzero-pressure-gradient cases.

\section{Description of the method}

The present paper shows the possibility to extrapolate $\left\{u_{\tau}, \Delta y, \kappa, \Pi, \delta\right\}$ from one measured experimental velocity profile. Here, the thickness of the boundary layer, $\delta$, is defined as the point where the mathematical description of the velocity profile reaches asymptotically the freestream velocity, $\left.u^{+}\right|_{y=\delta}=u_{e}^{+}$and $\left.\frac{\mathrm{d} u^{+}}{\mathrm{d} y^{+}}\right|_{y=\delta}=0$. In order to obtain these values, one can define a canonical description of the mean velocity profile, which depends on the aforementioned parameters, and then compare with the experimental mean velocity profile. The set of five variables, which minimize the error with respect to the canonical description to the experimental velocity profile, is taken as the optimal solution of the method.

Allowing a possible offset to the wall location will account for the uncertainties in the relative position of the measurement device with respect to the wall. Let $y^{\star}$ be the 
estimated wall normal positions at which the experimental velocity profile is measured. The uncertainty associated with the wall location can be just taken as an additive constant $\Delta y$ defining the wall normal location in inner variables as $y^{+}=\left(y^{\star}+\Delta y\right) u_{\tau} / v$, which depends explicitly on $u_{\tau}$ and $\Delta y$. Let us define $u^{\star}\left(y^{\star}\right)$ as the mean velocity measured for every wall normal location $y^{\star}$ defining therefore $u_{e x p}^{+}=u^{\star} / u_{\tau}$, which obviously only depends on $u_{\tau}$.

The canonical description of the boundary layer can be made as follows. One can assume that the inner layer $\left(y^{+}<5\right)$ is described by $u^{+}=y^{+}$. Assuming the validity of the logarithmic law verifies $u^{+}=\frac{1}{\kappa} \ln \left(y^{+}\right)+B$ in which, as we have justified in the previous section, the von Kármán constant is a free parameter in the optimization. A continuous description of the buffer layer adapting the inner and logarithmic layers is given by Musker (1979) as:

$\frac{d u^{+}}{d y^{+}}=\frac{\frac{\left(y^{+}\right)^{2}}{\kappa}+\frac{1}{s}}{\left(y^{+}\right)^{3}+\frac{\left(y^{+}\right)^{2}}{\kappa}+\frac{1}{s}}$

where $s$ is a constant that relates the eddy viscosity in the inner and overlap regions. This formulation is commonly accepted (Kendall and Koochesfahani 2008; Chauhan et al. 2007; Orlu et al. 2010) and has been improved by the inclusion on the formulation of an overshoot over the logarithmic law on the buffer layer, first reported by Monkewitz et al. (2007) to reflect experimental and numerical evidence, in the form of

$u_{\text {bump }}^{+}=\frac{\exp \left[-\log ^{2}\left(y^{+} / M_{1}\right)\right]}{M_{2}}$

with $M_{1}=30$ and $M_{2}=2.85$. Note that the inclusion of this overshoot over the logarithmic law achieves a better description of the mean velocity profile but, contrary to the description of Musker (1979) (based on eddy viscosity hypothesis), is not deduced from any equation and empirically fits high Reynolds number data. This is why two different canonical velocity profiles are subsequently used. Figure $1 \mathrm{~b}$ shows the variable $\Xi=u^{+}-\left[\frac{1}{\kappa} \ln \left(y^{+}\right)+B\right]$ where the overshoot described by Eq. 2 is clearly seen. Let us define the Musker velocity profile as that for which the overshoot is not considered and define the bump profile as that for which the overshoot over the logarithmic law is taken into account.

The role of the parameter $s$, as described in Musker (1979), has the effect of changing the constant of integration, $B$, in the logarithmic description of the boundary layer. Making use of the same argument as above, the overlap region (where $\frac{\mathrm{d} u^{+}}{\mathrm{d} y^{+}}=\frac{1}{\kappa y^{+}}$) just provides information about the slope of the logarithmic law, not about its integration constant. Nagib and Chauhan (2008), by plotting several values of the constants $\kappa$ and $B$ for different boundary conditions and pressure gradients, found an empirical collapse of the data over the curve described by:

$\kappa B=1.6[\exp (0.1663 B)-1]$

Equation 3 is also supported by the study of Vinuesa (2013) who extrapolates the wall location as that which minimizes the residual between the optimized pair $(\kappa, B)$ and the curve given by Eq. 3. Another expression that could close the problem would be $\kappa B=B\left(u_{\log }^{+}-B\right)^{-1} \ln y_{\log }^{+}$(obtained by rearranging the log-law expression). This equation has the advantage of not being based on experimental results. However, it is only valid for the pair $\left(u_{\log }^{+}, y_{\log }^{+}\right)$belonging to the logarithmic layer and thus requires the a priori specification of the limits of the logarithmic layer. Optimizations using this equation have been taken, and the results (not shown for brevity) show that the method fails to reproduce the friction velocity when applied to low Reynolds number or nonzero-pressure gradient. Therefore, the present paper will assume the relation $B=B(\kappa)$ or, equivalently, $s=s(\kappa)$ given by Eq. 3, so this parameter will be dependent on the von Kármán constant.

The outer part of the boundary layer will be taken after the description given by Coles (1956) where the wake will be defined as the exponential wake function described in Chauhan et al. (2009). The canonical description of the boundary layer is therefore given by:

$u_{\text {canonical }}^{+}= \begin{cases}u_{\text {musker }}^{+}+u_{\text {bump }}^{+}+\frac{2 \Pi}{\kappa} \mathcal{W}(y / \delta) & 0 \leq y \leq \delta \\ u_{e}^{+} & \delta \leq y \leq \infty\end{cases}$

where $u_{\text {musker }}^{+}$is given by the integration of Eq. 1 by an Euler method marching in $y$ from $u^{+}=0$ for $y^{+}=0$ with a step on $y^{+}$of 0.001. $u_{\text {bump }}^{+}$is given by Eq. 2 and can be activated (bump profile) or deactivated (Musker profile). $\mathcal{W}(y / \delta)$ is described in Chauhan et al. (2009) and is given by:

$$
\begin{aligned}
\mathcal{W}(\eta)= & \frac{1-\exp \left[-(1 / 4)\left(5 a_{2}+6 a_{3}+7 a_{4}\right) \eta^{4}+a_{2} \eta^{5}+a_{3} \eta^{6}+a_{4} \eta^{7}\right]}{1-\exp \left[-(1 / 4)\left(a_{2}+2 a_{3}+3 a_{4}\right)\right]} \\
& \times\left(1-\frac{1}{2 \Pi} \ln (\eta)\right)
\end{aligned}
$$

where $\quad \eta=y / \delta, a_{2}=132.8410, a_{3}=-166.2041$, and $a_{4}=71.9114$. Note that the canonical velocity profile is defined as a function of just $\{\kappa, s(\kappa), \Pi, \delta, y\}$. In order to compare with the experimental velocity profile, it has to be defined for the points in which the velocity is measured, $y^{\star}$. This introduces the dependence of the friction velocity and the wall offset. Therefore, $u_{\text {canonical }}^{+}$depends on $\left\{u_{\tau}, \Delta y, \kappa, \Pi, \delta\right\}$. 
We can define the residual error of the optimization method, $E$, in two ways. First, $E_{1}$ is taken as the mean relative error, it will give greater weighting to the points close to the wall where the velocity is smaller. This is done in order to enhance the sensitivity of the method to these points, which provide more important information about the inner scaling and the friction velocity. The second method, $E_{2}$, will be defined as the mean quadratic error, considering all the points of the velocity profile with the same importance. The two definitions of the error are thus

$E_{1}\left(u_{\tau}, \Delta y, \kappa, \Pi, \delta\right)=\left\langle\frac{\left|u_{\text {canonical }}^{+}\left(u_{\tau}, \Delta y, \kappa, \Pi, \delta\right)-u_{\text {exp }}^{+}\left(u_{\tau}\right)\right|}{u_{\text {canonical }}^{+}\left(u_{\tau}, \Delta y, \kappa, \Pi, \delta\right)}\right\rangle$

$E_{2}\left(u_{\tau}, \Delta y, \kappa, \Pi, \delta\right)=\left\langle\sqrt{\left(u_{\text {canonical }}^{+}\left(u_{\tau}, \Delta y, \kappa, \Pi, \delta\right)-u_{\text {exp }}^{+}\left(u_{\tau}\right)\right)^{2}}\right\rangle$

Thus, the constrained optimization problem results as follows, with the exact values provided in Table 2 .

find $\min \left[E\left(u_{\tau}, \Delta y, \kappa, \Pi, \delta\right)\right]$
subject to $\left\{\begin{aligned} u_{\tau} & \in\left[u_{\tau, \min }, u_{\tau, \max }\right] \\ \Delta y & \in\left[\Delta y_{\min }, \Delta y_{\min }\right] \\ \kappa & \in\left[\kappa_{\min }, \kappa_{\min }\right] \\ \Pi & \in\left[\Pi_{\min }, \Pi_{\max }\right] \\ \delta & \in\left[\delta_{\min }, \delta_{\max }\right]\end{aligned}\right.$

The initial conditions have been selected as typical and possible reasonable values for the different variables of the optimization. In any case, the problem is convex and therefore independent of the initial conditions but faster convergence is found if they are chosen according to the sensible estimations of the variables. The bounds of the optimization are activated in order to ensure robustness in the method. They are selected to be far enough from any optimal point so as to not have any influence on the results. Different bounds have been tested without effect either on the result of the method, or on the convergence rate.

Let us define the Lagrangian of the problem as $\mathcal{L}\left(\boldsymbol{x}_{k}, \lambda\right)=E\left(x_{k}\right)-\boldsymbol{\lambda}^{T} \boldsymbol{b}\left(\boldsymbol{x}_{k}\right)$, where the error function is $E: \mathbf{R}^{5} \longmapsto \mathbf{R}$, defined in Eqs. 6 or $7, \boldsymbol{b}$ comprises the bounds of the optimization expressed as $\boldsymbol{b}(\boldsymbol{x}) \geq 0$, and $\boldsymbol{\lambda}$

Table 2 Upper and lower limits and starting point of the optimization

\begin{tabular}{llllll}
\hline & $u_{\tau}$ & $\Delta y$ & $\kappa$ & $\Pi$ & $\delta$ \\
\hline Max & $1.5 \tilde{u}_{\tau}^{\mathrm{a}}$ & $-y_{\text {ini }}^{\star}$ & 0.8 & 5 & $8 \delta_{99}$ \\
Min & $0.5 \tilde{u}_{\tau}^{\mathrm{a}}$ & $\infty$ & 0.2 & 0 & $0.8 \delta_{99}$ \\
Initial & $\tilde{u}_{\tau}^{\mathrm{a}}$ & 0 & 0.4 & 0.5 & $1.3 \delta_{99}$ \\
\hline
\end{tabular}

a $\tilde{u}_{\tau}$ is estimated by the Clauser (1954) method

b $y_{i n i}^{\star}$ is the measured location of the first point are the Lagrange multiplicators. The problem is solved using sequential quadratic programming (Nocedal and Wright 2006), that is, for a given $\boldsymbol{x}_{\boldsymbol{k}}$, the Lagrangian, $\mathcal{L}$, of the system is approached by a quadratic subproblem, and then, the search direction, $\boldsymbol{d}_{\boldsymbol{k}}$, is found as the solution of the following subproblem:

$$
\begin{gathered}
\text { find } \min _{d}\left[E\left(\boldsymbol{x}_{\boldsymbol{k}}\right)+\nabla f\left(\boldsymbol{x}_{\boldsymbol{k}}\right)^{T} \boldsymbol{d}+\frac{1}{2} \boldsymbol{d}^{T} \nabla_{x x}^{2} \mathcal{L} \boldsymbol{d}\right] \\
\text { s.t. } \boldsymbol{b}\left(\boldsymbol{x}_{\boldsymbol{k}}\right)+\nabla \boldsymbol{b}\left(\boldsymbol{x}_{\boldsymbol{k}}\right)^{T} \boldsymbol{d} \geq 0
\end{gathered}
$$

approaching the derivatives by centered finite differences in second order. Once the search direction is known, the next step of the optimization will be $\boldsymbol{x}_{\boldsymbol{k}+\boldsymbol{1}}=\boldsymbol{x}_{\boldsymbol{k}}+\alpha \boldsymbol{d}_{\boldsymbol{k}}$, where $\alpha$ is a constant. Note that in the present problem, $\boldsymbol{x}_{\boldsymbol{k}}, \boldsymbol{d}_{\boldsymbol{k}} \in \mathbf{R}^{5}$. The tolerances in $\boldsymbol{x}_{\boldsymbol{k}}$ and in the function $E$ are set to $10^{-10}$, the tolerance in the constraint violation is relaxed to $10^{-6}$ since the constrains do not play any important role in the optimization problem.

\section{Results}

The aim of this section was to provide information about the accuracy and robustness of the proposed method. The topology of the error function in the vicinity of the optimal point will be shown in order to justify that the method is absolutely convex and with a single solution. The next two subsections will compare the results obtained by the method with flows in which the friction coefficient is independently measured, i.e., the experiments of Österlund (1999) in zero-pressure-gradient turbulent boundary layers and high Reynolds number DNS performed by Jiménez (2014) [fully documented in Sillero et al. (2013); Simens et al. (2009); Borrell et al. (2013)] and Schlatter (2014) [fully documented in Schlatter et al. (2009); Schlatter and Örlü (2010)]. The next sections will study the importance of the location of the first point with respect to the wall and a robustness study to various perturbations. The last part will provide a discussion of the results as well as some points to consider in advance of performing experiments if this method is to be used in the post-processing.

Results are presented for the four methods studied, that is, activating or not the bump on the velocity profile given by Eq. 2, and using both possible definitions of the error given by Eqs. 6 and 7, we can therefore define the methods bump1, bump2, musker1, and musker 2 , respectively.

\subsection{Error topology}

As stated above, the use of the von Kármán constant as an optimization parameter is justified based on the existent overlap between inner and outer layers, which is seen by the flow as a change in the slope of the adaptation region. 
However, one may realize that the change that the velocity profile (Eq. 4) experiences for a given change in $\kappa$ is similar to the change that it would experience for a change in $u_{\tau}$. This is the cause of the linear relation present in the residual function $E\left(\kappa, u_{\tau}\right)$ (Fig 2a-d). This ambiguity, which in fact is present in the Clauser (1954) method, does not appear in the present method, since the measurements out of the log layer introduce further information, generating a clearly defined minimum in the vicinity of the optimum for every method tested. This can be seen in Fig. 2, where a clear convex topology is shown along with the fact that the method, independently of which one is used, detects this minimum.

Regarding the error function $E: \mathbf{R}^{5} \longmapsto \mathbf{R}$, it cannot be represented in two dimensions; therefore, we define

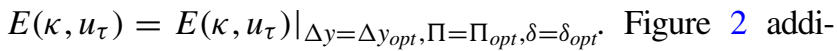
tionally shows that the sensitivity of the method to changes in the friction velocity is greater than the sensitivity on the other variables of the optimization such as $\kappa$ or $\Delta y$ as previously reported by Kendall and Koochesfahani (2008). This makes it possible for the method to overcome some uncertainties on the other variables but still provide an accurate estimation of $\tau_{\text {wall }}$.

\subsection{Validation with Österlund database}

In order to validate the current approach, the results obtained by the present method are compared with the
Österlund (1999) database. It contains data taken using hot wire anemometry over a zero-pressure gradient turbulent boundary layer, and it has been used as a benchmark for testing different methods to determine $\tau_{\text {wall }}$ (Kendall and Koochesfahani 2008; Chauhan et al. 2007; Nagib et al. 2007). The four methods proposed (bump1, bump2, musker1, musker2) are tested for the 70 velocity profiles $2533<R e_{\theta}<27,320$ making a total of 280 validation cases. The friction velocity is independently measured using oil film interferometry. Fernholz et al. (1996) report that usually the accuracy of this method is $\pm 4 \%$, further improvements to the method ensure that it can be taken as $\pm 1.5 \%$ (Nagib et al. 2004), this is the error assumed in the measurements by Österlund (1999). The probes are positioned with respect to the wall using a microscope with accuracy of $\pm 5 \mu \mathrm{m}$ (Österlund 1999).

Let us define $\epsilon(\%)=\frac{u_{\tau}^{\text {opt }}-u_{\tau}^{\text {measured }}}{u_{\tau}^{\text {measured }}} \times 100$ as a measure of the error that the present method introduces on the determination of the friction velocity. The velocity $u_{\tau}^{\text {measured }}$ is taken as the result of the independent measures performed by Österlund (1999). Note that the possible error on the determination of $u_{\tau}^{\text {measured }}$ is not considered for the definition of $\epsilon$.

$E_{o p t}$ is defined as the value of the optimization function (Eqs. 6 or 7) at the optimal point. It is obvious from

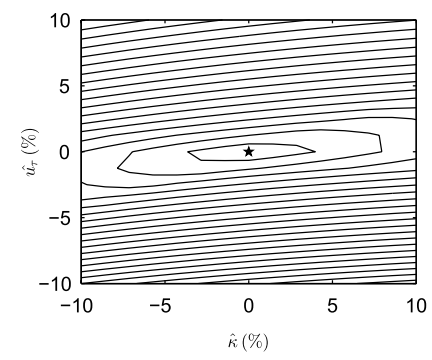

(a)

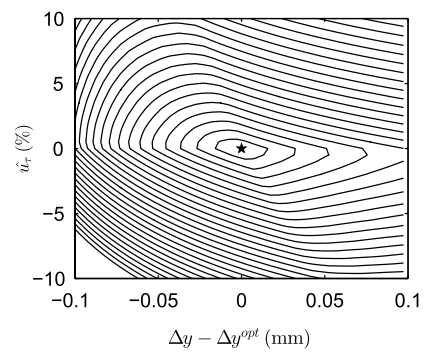

(e)

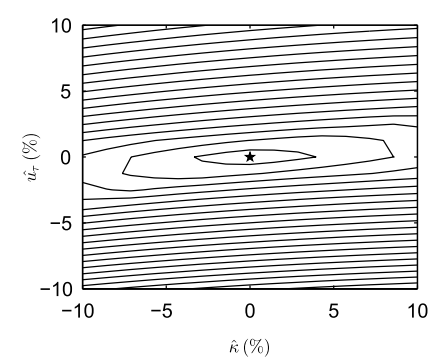

(b)

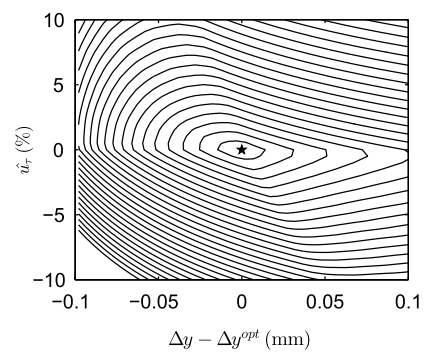

(f)

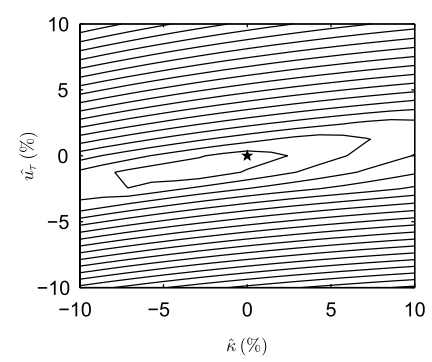

(c)

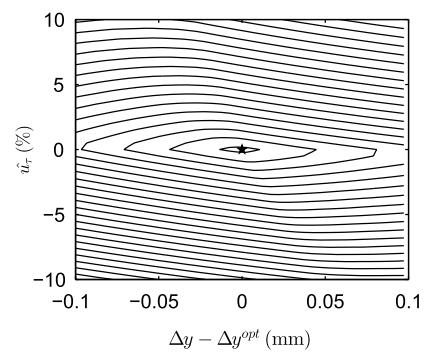

(g)

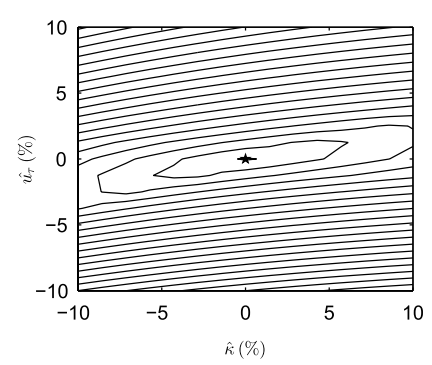

(d)

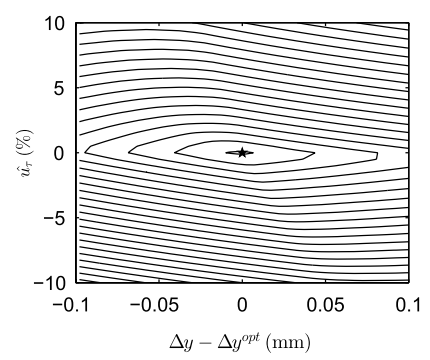

(h)

Fig. 2 Topology of the error $E_{1,2}$ in the vicinity of the optimum. Dataset SW981129A of Österlund (1999). $\hat{u_{\tau}}=\frac{u_{\tau}-u_{\tau}^{o p t}}{u_{\tau}^{o t}} \in[-10,10 \%]$ and $\hat{\kappa}=\frac{\kappa-\kappa^{o p t}}{\kappa^{o p t}} \in[-10,10 \%]$ Isolevels for $E_{1}(\mathbf{a}, \mathbf{b}, \mathbf{e}, \mathbf{f})$ are from 0.009 closest to the optimum increasing

in steps of 0.007. Isolevels for $E_{2}(\mathbf{c}, \mathbf{d}, \mathbf{g}, \mathbf{h})$ are from 0.06 closest to the optimum increasing in steps of 0.12 . Black star shows the optimal point found by the optimization method 


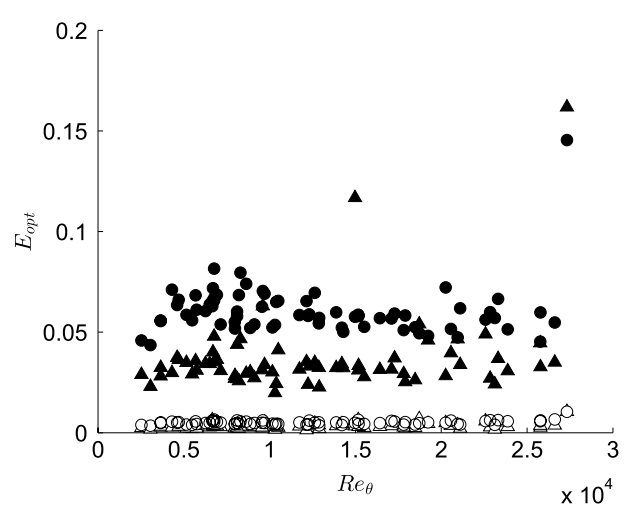

(a)

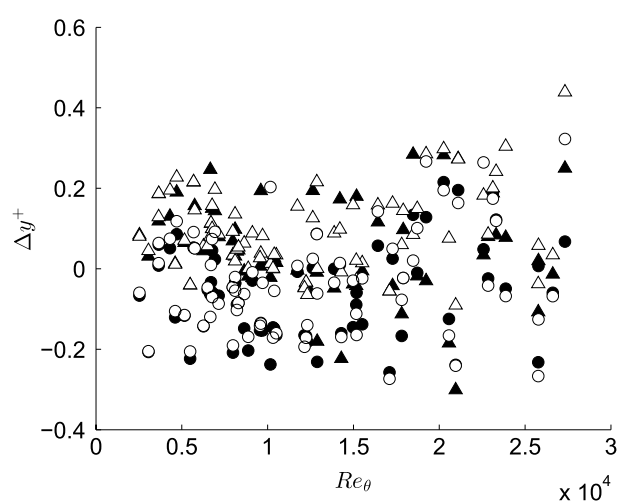

(c)

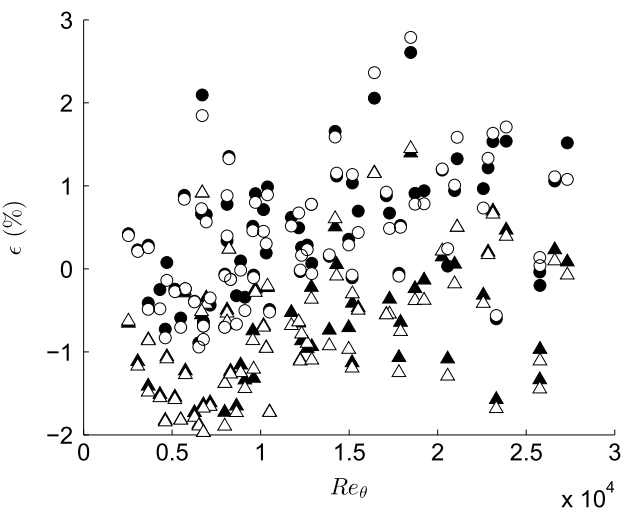

(b)

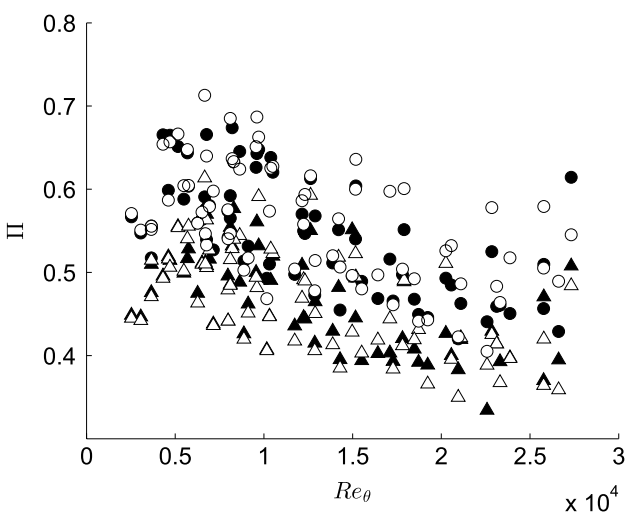

(d)

Fig. 3 Variation in optimization parameters with $R_{\theta} .4$ methods $\times 70$ Österlund (1999) data sets $=280$ validations. Symbols mean: open triangle bump1, filled triangle bump2, open circle musker1, filled circle musker 2 .

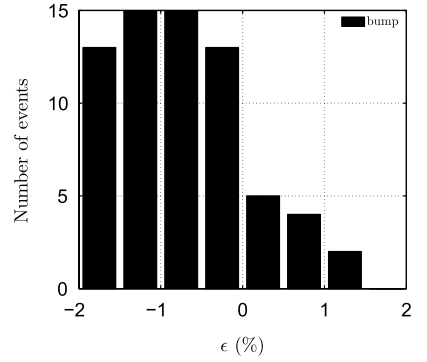

(a)

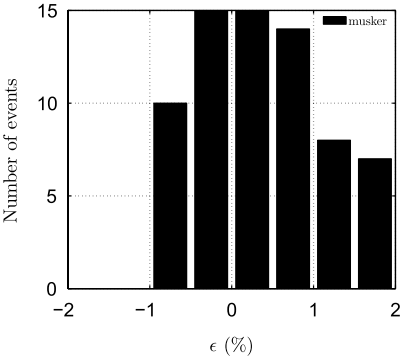

(b)

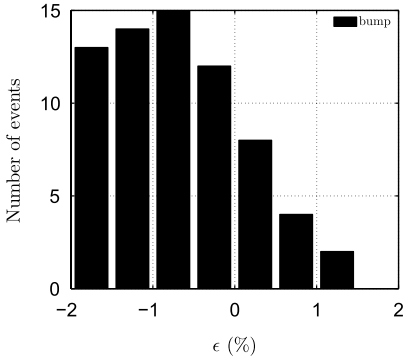

(c)

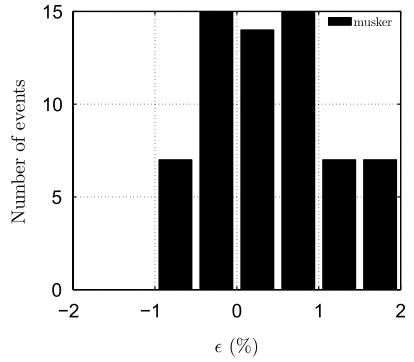

(d)

Fig. 4 Histograms of error, $\epsilon$, for validation with 70 data sets of Österlund (1999). $\mathbf{a} \epsilon$ for profile bumpl, $\mathbf{b} \epsilon$ for profile muskerl, $\mathbf{c} \epsilon$ for profile bump $2, \mathbf{d} \in$ for profile musker 2

the definition that $E_{2}>E_{1}$, this can be seen also in Fig. 3a. However, there are two clear behaviors of the value of the optimal residual $E_{\text {opt }}$. For every measured point $E_{\text {opt }}^{\text {bump }}<E_{\text {opt }}^{\text {musker }}$, Although this is not a surprising result (the expression of the bump is calculated to fit, among others, these experimental results), the opposite trend is followed by $|\epsilon|$. As one can see in Fig. 4, Musker profiles provide a centered error, which can be attributed to statistical dispersion. The bump profile, on the contrary, presents a biased error toward negative values. Although this error is consistently biased, its small magnitude $\left(\left\langle\epsilon_{1,2}^{\text {bump }}\right\rangle=-0.7 \%\right)$ always places it within the experimental error of the method. Note also that the biggest dispersions of $\epsilon$ using the bump profile are concentrated in the smallest Reynolds numbers. This is an expected result since the definition of the bump fits better high Reynolds number boundary layers, where one cannot see any difference between the use of the two different velocity profiles (Fig. 3b). 
With respect to the wall positioning, the method always provides $\Delta y^{+}<0.25$, which translated into physical units is approximately $\Delta y<5 \mu \mathrm{m}$, the diameter of a typical hot wire probe. It suggests that the actual accuracy of the method is higher and what we are detecting here may be the uncertainty present in the measurements of Österlund (1999), this effect will be further discussed in Sects. 3.3 and 3.4.

Regarding the value of the wake parameter, $\Pi$, the main result that can be observed is that for every experimental data set, $\Pi^{\text {bump }}>\Pi^{\text {musker }}$. Special care should be taken with this aspect; in particular, one has to be aware that, contrary to what happens with $u_{\tau}$, there is not a correct value for $\Pi$. The wake component provides a way to describe the velocity profile in a way that is dependent upon the fitting method and the canonical description chosen for the velocity profile. This makes it very difficult to compare with published results in which different definitions are taken. As one example of this, Chauhan et al. (2009) compute $\Pi$ with the same wake function but he explicitly decides not to include the formulation of the bump reasoning that a small change on the buffer region does not affect the outer part of the boundary layer. The results shown in Fig. 3d exhibit clear differences when including or not the bump in the optimization. This may be due to the inclusion in the optimization of $u_{\tau}$, which was not included by Chauhan et al. (2009) and links the behavior in the inner and outer layers. One has to ensure therefore that the same method is used to obtain the wake component if the results are going to be compared with published data.

\subsection{Validation with DNS database}

Some of the features that have been observed in the validation with experimental data are also seen in the validation against DNS. In particular, we observe again the dependence of the wake component on the chosen description of the velocity profile (Fig. 5d). It can be also observed that the bump profile fits better the numerical results (Fig. 5a), where $E_{\text {opt }}^{\text {bump }}<E_{\text {opt }}^{\text {musker }}$. However, the contrary trend is seen with respect to the ability of the method to recover the accurate friction velocity, $\left|\epsilon^{\text {bump }}\right|<\left|\epsilon^{\text {musker }}\right|$ in Fig. 5b. Note that in this case, there is not any error in the determination of $u_{\tau}$ from DNS; this, together with the fact that $E_{\text {opt }}^{\text {bump }}<E_{\text {opt }}^{\text {musker }}$ sustains the observation of a better result produced by the bump profile. It can be also clearly seen that the error $\epsilon$ is smaller when using the definition of $E_{1}$ given by Eq. 6 .

Regarding the ability of the method to recover the accurate wall position, we can see in Fig. 5c that the accuracy is more than one order of magnitude larger than in comparison with experimental results. In particular, the trend
Fig. 5 Variation in optimization parameters with $\operatorname{Re}_{\theta}$. Comparison using DNS by Jiménez (2014) and Schlatter (2014). Symbols mean open triangle bump1, filled triangle bump2, open circle muskerl, filled circle musker 2 . a $E_{\text {opt }}=f\left(\operatorname{Re}_{\theta}\right)$, $\mathbf{b} \epsilon=f\left(\operatorname{Re}_{\theta}\right), \mathbf{c} \Delta y^{+}=f\left(R_{\theta}\right)$, $\mathbf{d} \Pi=f\left(\operatorname{Re}_{\theta}\right)$

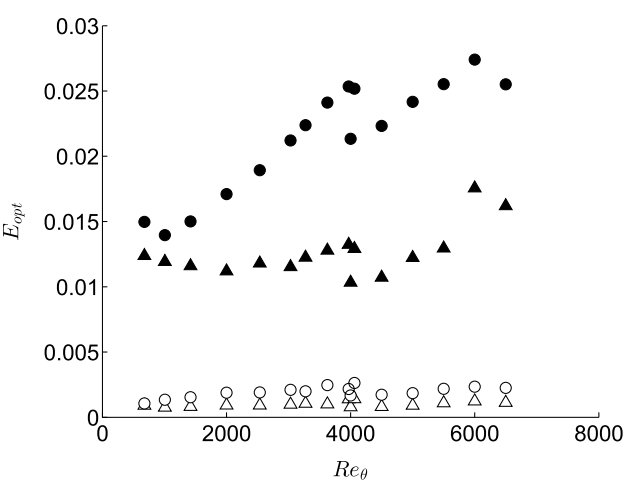

(a)

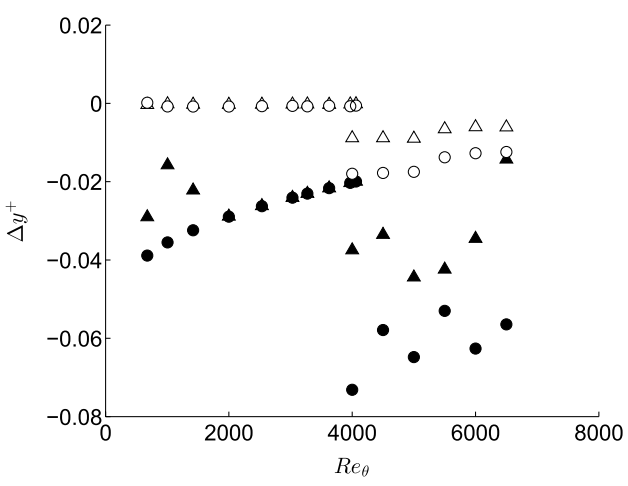

(c)

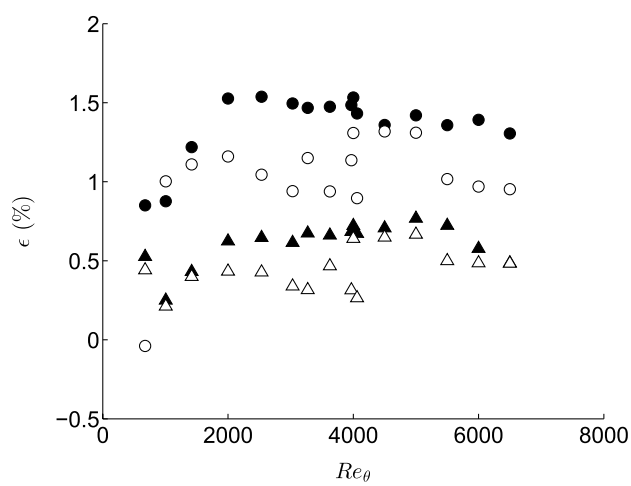

(b)

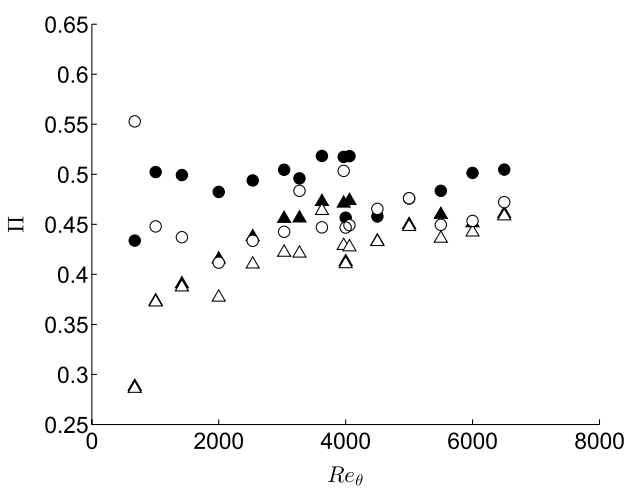

(d) 
followed by the comparison with DNS of Schlatter (2014), $\left(R e_{\theta}<4000\right)$ differs from the DNS by Jiménez (2014) $\left(R e_{\theta}>4000\right)$. This is because the distribution of the points in the near-wall region is different for both DNS (higher concentration of points for Schlatter (2014) results, which provide a more accurate wall detection). The decreasing trend followed by the error of $\Delta y$ for increasing Reynolds number is justified by the distribution of points in the nearwall region for the computational domains. The turbulent boundary layer is evolving in a domain where the grid points have a constant distance from the wall in physical units, it means that a larger number of points is included in the near-wall region for high Reynolds number. This fact shows the importance of the points that are located in the near-wall region, their separation, and their initial position, and this effect will be further studied on the next section.

\subsection{Influence of the first point, $y_{0}^{+}$}

One of the sources of differences between the results shown in Sects. 3.3 and 3.2 is the location of the first point. In the case of DNS, the first point is located at $y_{0}^{+} \sim 0.01$, whereas the experiments are taken with the first point measured at between 4 and 5 wall units. Orlu et al. (2010) studied the influence of the location of the first point and concluded that the closer to the wall this point is located, the smaller the errors of the method. Similar results are shown in this study where, as shown in Fig. 6, the errors increase with increasing distance of the first point from the wall. In particular, we can see that taking the first point at $y_{0}^{+}=10$ provides an error in the wall offset of $\Delta y^{+}<0.5$. Note also that the sensitivity to the bump method is much smaller than Musker, where an abrupt change in the values is reported for $y_{0}^{+}>10$. Figure 6 can be used by future experimentalists to provide information on the expected accuracy of the method as a function of the location of the closest point to the wall. This accuracy will be higher the closer the points can be measured. Although it will only be known after the performance of the experiment, an estimation of $\Delta y^{+} \sim 0.3$ for the commonly available positioning methods may be appropriate.

\subsection{Influence of distorted wake region. Pressure gradient}

In order to validate the method for non-canonical boundary layer flows, it will be tested against the Watmuff (2014) database of turbulent boundary layers affected by both adverse and favorable pressure gradients covering $368 \leq R e_{\theta} \leq 4857$, fully documented in Watmuff (1990). This study presents measurements of the wall shear stress by means of Preston tubes of different diameters, and due to the experimental techniques, the uncertainty in the determination of $C_{f}$ is expected to be bigger than in the Österlund (1999) database. Watmuff (1990) estimates it as $\pm 3 \%$. Figure 7 a shows that the present method succeeds in the determination of the friction velocity within the quoted range of uncertainty. Figure $7 \mathrm{~b}$ shows that it also recovers the exact wall location with an error $\left|\Delta y^{+}\right| \leq 0.5$ for medium values of the pressure gradient coefficient, $\beta=\frac{\delta_{\star}}{\tau_{w}} \frac{\mathrm{d} p}{\mathrm{~d} x}$, and $\Delta y^{+} \sim \pm 1$ for $\beta>3$. This deviation corresponds with the uncertainty reported by Watmuff (1990), $50 \mu \mathrm{m}$, which corresponds to $0.4 \leq \Delta y^{+} \leq 1.3$ for the different friction velocities. Two particular features are seen; first, as reported in previous sections, using the definition of the residual error $E_{1}$ given by Eq. 6 provides a better result when determining the absolute wall position. Second, contrary to previous sections, the estimation of the wall shear stress is more accurate when using the Musker velocity profile than when including also the bump in the

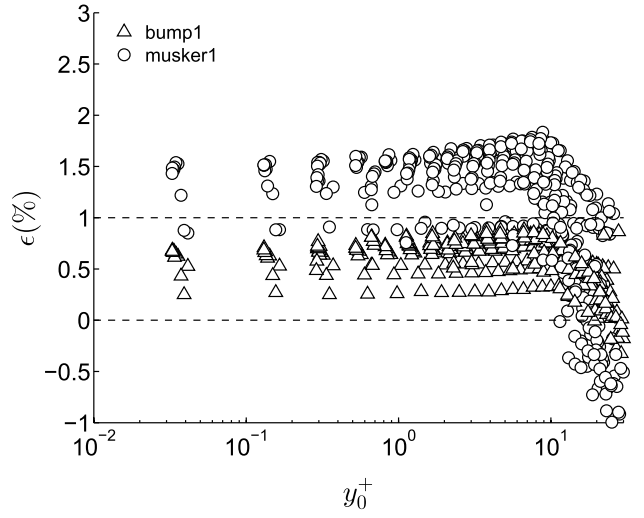

(a)

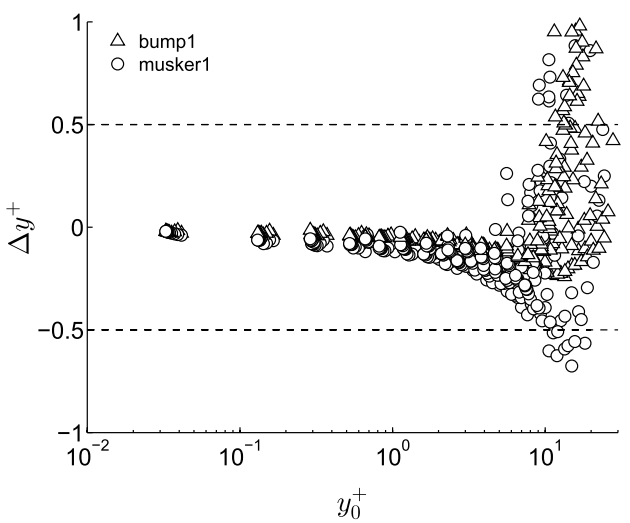

(b)

Fig. 6 Variation in optimization parameters with $y_{0}^{+}$, the location of the closest point to the wall. Comparison using DNS by Jiménez (2014) and Schlatter (2014), $\mathbf{a} \epsilon=f\left(y_{0}^{+}\right), \mathbf{b} \Delta y=f\left(y_{0}^{+}\right)$ 


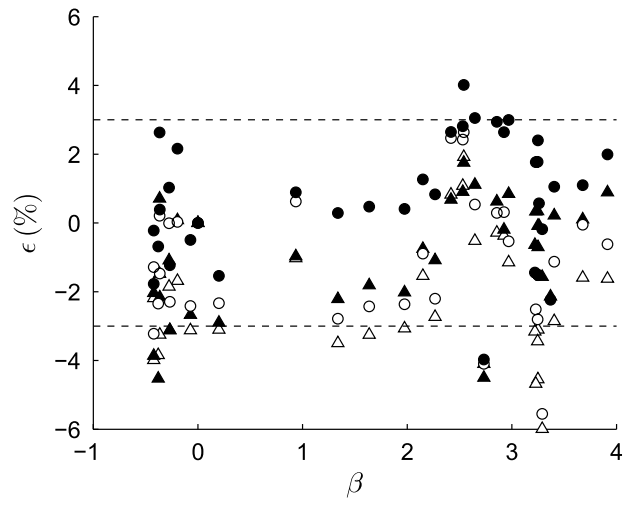

(a)

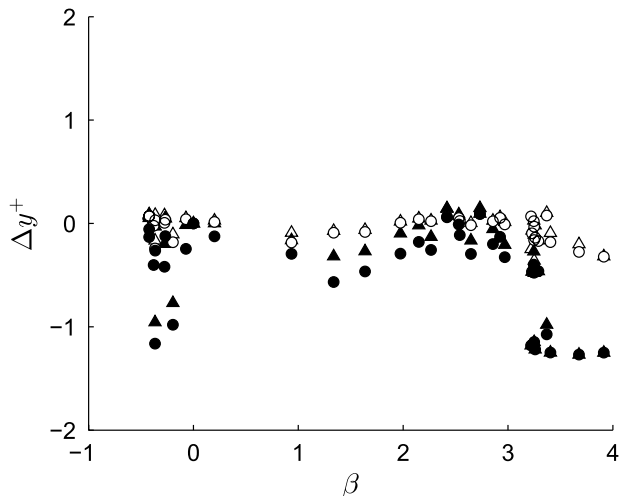

(b)

Fig. 7 Variation in optimization parameters with $\beta=\frac{\delta_{\star}}{\tau_{w}} \frac{\mathrm{d} p}{\mathrm{~d} x}$, the pressure gradient coefficient. Comparison using Watmuff $(2014)$ database. Symbols mean open triangle bump1, filled triangle bump2,

open circle musker1, filled circle musker 2 . Dashed line marks $\pm 3 \%$ , the estimated accuracy on the determination of $C_{f}$. $\mathbf{a} \epsilon=f(\beta), \mathbf{b}$ $\Delta y=f(\beta)$
Fig. 8 Variation in optimization parameters with the level of noise in the velocity measurements, $\xi$. Comparison using DNS $\operatorname{Re}_{\theta}=6500$ of Jiménez (2014). Symbols mean open triangle bump1, filled triangle bump2, open circle musker1, filled circle musker $2 . \mathbf{a} \epsilon=f(\xi)$ b $\Delta y=f(\xi)$

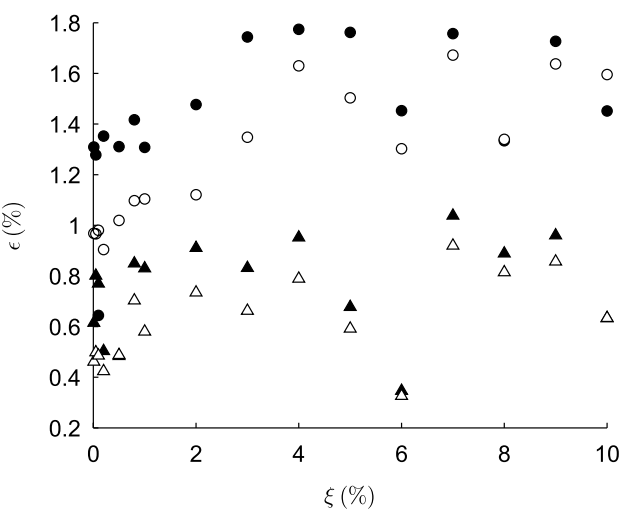

(a)

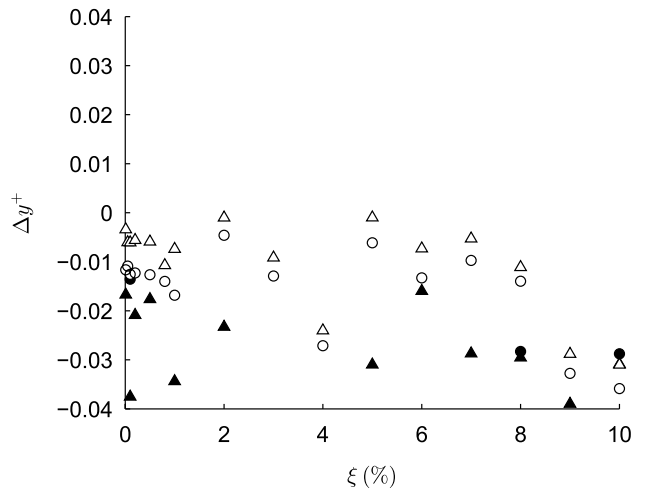

(b) formulation. This may be because the pressure gradient affects the natural development of the boundary layer making the definition of the bump (developed for zero-pressure gradient) not valid. Note that these results are tested for relatively mild pressure gradients $\left(-0.009 \leq p_{x}^{+} \leq 0.02\right)$ where $\kappa$ is not expected to present high variations. However, they have been obtained without any a priori specification of the von Kármán constant, which is known to be influenced by the pressure gradient (Nickels 2004; Nagib and Chauhan 2008). It also supports the use of Eq. 3 for flows influenced by different pressure gradients.

\subsection{Influence of velocity measurements. Gaussian noise}

In order to assess the robustness of the method with respect to statistical errors caused by the velocity measurements, we test one of the DNS by Jiménez (2014), $R e_{\theta}=6500$, applying a Gaussian noise with zero mean and amplitude proportional to the turbulence intensity for every point of the velocity profile. This tries to simulate a poor convergence of the velocity statistics due to insufficient sampling time. The new velocity is obtained as

$u_{\text {noise }}(y)=u(y)+N\left(0, \xi \cdot u^{\prime}(y)\right)$

where $N(\mu, \sigma)$ is a random Gaussian variable of mean $\mu$ and variance $\sigma, u^{\prime}$ is the turbulence intensity and $\xi \in[0.01,10 \%]$. In order not to contaminate the results due to the addition of noise, with those uncertainties associated with the location of the first measurement point, or the number of points of the profile (covered in Sects. 3.4 and 3.7), the whole mean velocity profile is used. Figure 8 shows that the method maintains its accuracy up to the highest $\xi=10 \%$ tested whether we use the bump formulation. On the contrary, the accuracy of the estimation of the wall shear stress is clearly affected by the noise level if one does not consider this overshoot. This may be due to the high turbulence intensity in the buffer layer, where the bump is located, which may distort more the measurements in this zone. Note also that the inner points, which provide most of the relevant information for the wall shear stress 


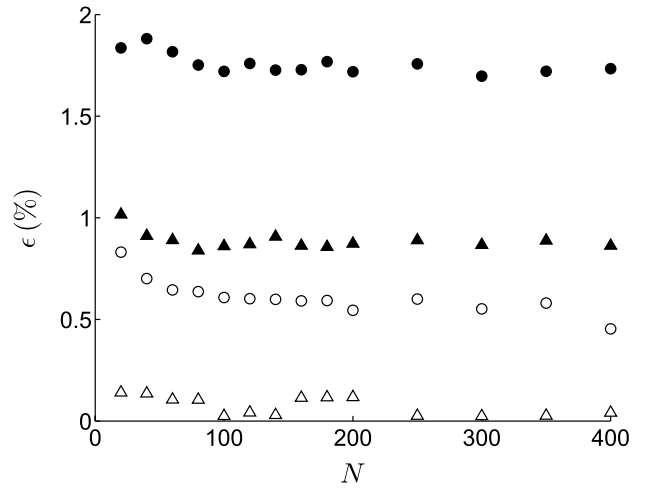

(a)

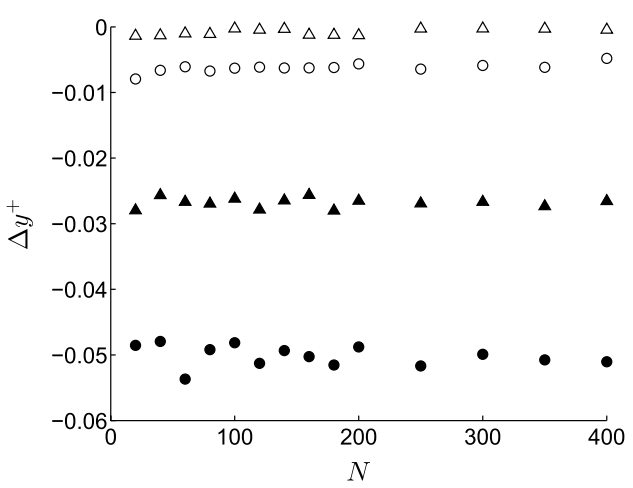

(b)

Fig. 9 Variation in optimization parameters with the number of points inside the boundary layer, $N$. Comparison using DNS $R e_{\theta}=6500$ of Jiménez (2014). Symbols mean open triangle bump1, filled triangle bump2, open circle musker 1 , filled circle musker $2 . \mathbf{a} \epsilon=f(N)$, b $\Delta y=f(N)$

(as will be shown in Sect. 3.7), are less affected by this kind of Gaussian noise, since the fluctuations in the viscous sublayer tend to zero near the wall; this may explain why the error definition $E_{1}$ shows a clearly better result because of the greater weighting of the near-wall points. A different kind of perturbation will be studied in Sect. 3.8 where these effects are assessed.

\subsection{Influence of the number of points}

The number of points in the velocity profile is a parameter of a particular experiment, which has to be decided $a$ priori. This section aims to provide an estimation of the number of points needed to achieve sufficient resolution for the extrapolation of the friction velocity. A common practice is to choose the measurement points logarithmically, which ensures that more velocity readings are taken in the inner region of the boundary layer. The velocity profiles used in this section will be obtained by undersampling the highest Reynolds number dataset by Jiménez (2014) logarithmically with $18 \leq N \leq 371$ points inside the boundary layer (i.e., for $y<\delta$ ). The small separation between the original cells of the DNS makes it possible to use linear interpolation when the desired wall normal location is not found. Figure 9 shows that the accuracy of the method is not affected by the number of points if we use the bumpl method up to the number of points simulated. As expected, using the residual error definition $E_{1}$ provides a significantly better result since it applies greater weighting to points located close to the wall. If, on the contrary, a linear scheme is used to under-sample the velocity profile, the results are seen to be greatly affected if the number of points inside the boundary layer is $<50$ (meaning between 4 and 7 points in the logarithmic region). While assessing the influence of the number of points, the location of the first point has been kept the same as in the original DNS.
The great difference presented for the residual definitions $E_{1}$ and $E_{2}$ and the study developed in Sect. 3.4 show the importance that the inner points have in the determination of the wall shear stress.

\subsection{Influence of distorted inner region. Probe interference}

In the last few years, the development of the diagnostic plot (Alfredsson and Örlü 2010) allows the experimentalist to spot points in the inner or viscous region, which have been influenced by the velocity probe, due to a local blockage or a significant heat conduction toward the wall in the case of hot wires. Both cases result in artificially high-velocity readings. The diagnostic plot presents the advantage of not needing either the friction velocity or the exact wall location in order to detect the distorted points. These points can be identified by their departure from the linear trend between $u^{\prime}$ and $u$ close to the wall, where turbulence fluctuations should decrease linearly with mean velocity. These deviations from the canonical profile have been simulated by modifying the inner points in the highest Reynolds number DNS of Jiménez (2014). Different intensities of distortion, $0 \leq \gamma \leq 30$, are tested for the points located in $3 \leq y^{+} \leq 8$ with shades spanning from black to white in Fig. 10a. The rest of the points of the velocity profile are kept unaltered. Although the uncertainty in $\Delta y^{+}$is affected by distorted points in the inner region (Fig. 11b), it is kept in reasonable values $\left(\Delta y^{+} \sim 1\right)$ up to stronger distortions than the ones expected in real experiments. Since the wall position presents a higher error, the estimated friction velocity tends to decrease (the similar trend is shown in Fig. 6). Note also that the determination of $\Delta y^{+}$is better when using the residual definition $E_{2}$, which gives smaller weighting to points in the inner region, which in this case are distorted by the velocity probe. Figure 11a shows that 


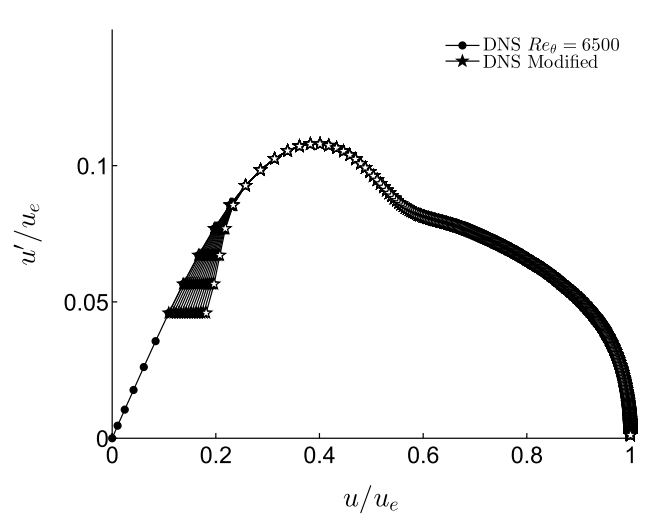

(a)

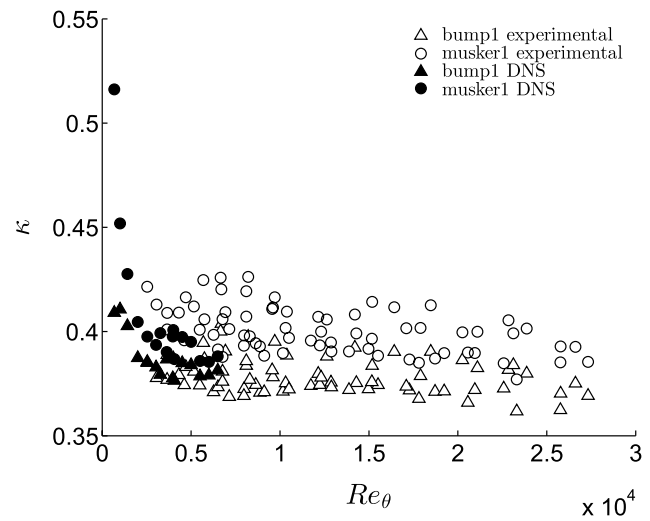

(b)
Fig. 10 a Diagnosis plot (Alfredsson and Örlü 2010) filled circle showing distorted inner regions $(\star)$. b Variations in von Kármán constant with $\operatorname{Re}_{\theta}$ using experimental data by Österlund (1999) (empty symbols) and DNS by Jiménez (2014) and Schlatter (2014) (filled symbols). a Diagnosis plot, $\mathbf{b} \kappa=f\left(\operatorname{Re}_{\theta}\right)$
Fig. 11 Variation in optimization parameters with the degree of distortion in the inner region, $\gamma$. Comparison using DNS

$\operatorname{Re}_{\theta}=6500$ of Jiménez (2014). Symbols mean open triangle bump1, filled triangle bump2, open circle muskerl, open circle musker $2 . \mathbf{a} \epsilon=f(\gamma), \mathbf{b}$ $\Delta y=f(\gamma)$

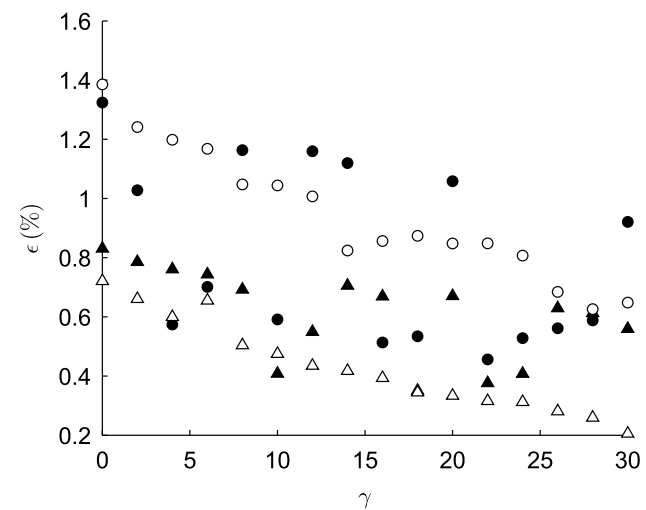

(a)

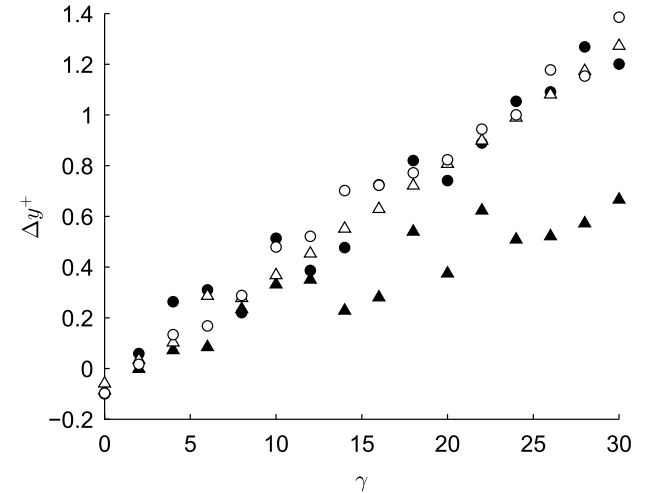

(b) globally the method presents a reasonable accuracy up to the highest distortion simulated, which is expected to be significantly stronger than the ones present in real experiments. The inaccuracy shown in $\epsilon=0.7 \%$ for $\gamma=0$ corresponds to the one due to the position of the first measurement point $y_{0}^{+}=3$ as studied in Sect. 3.4.

\subsection{Discussion}

Figure 10b shows that even though no further conditions have been imposed to the value of the von Kármán constant, it takes values similar to those already presented in the literature, $\kappa \in[0.37,0.45]$. This result provides further evidence of the used theory, namely one does not have to specify a given $\kappa$ based on the published data, but one can leave the optimization free to adopt a value of the von Kármán constant as a function of different boundary or initial conditions. On the other hand, there is clear dependence seen on the velocity profile used for the canonical description. In particular, using bump profile, the mean and standard deviation of the von Kármán constant are $\kappa=0.379 \pm 0.008$, whereas when using Musker profile, $\kappa=0.400 \pm 0.011$. Two main conclusions can be made. In the first place, it may explain why the value suggested by Nagib and Chauhan (2008) is $\kappa=0.384$, since the authors of this paper considered the bump to account for the overshoot present over the log layer in order to estimate the von Kármán constant. Some of the previous publications (where the experimental and numerical techniques were not as accurate as in the present and therefore did not report the appearance of the bump) seems to point closer to 0.4 or 0.41 . Secondly, we have to be especially careful when comparing results with published data. The sensitivity of the method is high enough so as to recognize differences in the used velocity profile and change the value of the von Kármán constant accordingly. This result also suggests a certain subjectivity on $\kappa=0.384$ published by Nagib and Chauhan (2008) since it is clearly shown that it presents a dependence on the velocity profile used. This is a further support for the present method where no subjective 
decisions have to be made regarding $\kappa$ in order to obtain the friction velocity. Finally, note that the aim and the main result of this paper are not simply to provide any value of $\kappa$. The main output of the present method should be seen as its ability to provide an accurate representation of the friction velocity $u_{\tau}$ and the wall position $\Delta y$ without any assumption a priori about the boundary conditions of the flow from the experimentally acquired velocity profile.

The importance of the first point has been assessed in Sect. 3.4. These results highlight the importance of being able to take velocity measurement as close as possible to the wall. Although the present method can locate with a high accuracy, the real position of the wall, it is still necessary to locate the velocity probes at $y^{+} \leq 10$ by other means before carrying out the measurements to ensure $|\epsilon|<1 \%$. The key difference with other methods applied in the past is that it may be sufficient to locate the probe close to the wall and start traversing it out from this location without knowing accurately the relative probe-wall position a priori. This is a considerable difference, which should make the experiments considerably easier to perform. In most of the cases, a simple camera placed close (but at an arbitrary angle) to the measurement point is accurate enough to avoid any damage to the probe but does not provide enough precision in the determination of the actual distance, which will be determined in the post-processing with the current method. Another advantage is that it does account for a possible deformation of the probe system under flow conditions, which may have not been taken into account in the determination of the wall position. The points close to the wall provide a big part of the information available for the method; as seen in Sects. 3.3 and 3.7 , the more points that are measured close to the wall, the smaller the uncertainty in the wall detection. However, before the performance of experiments, one has to consider that, as stated in Sect. 3.4, the location of the first point presents a higher significance than the number of points measured in the near-wall region for a more accurate estimation of wall stress. Consider also that the bump method provides a lower sensitivity than the Musker profile to the position of the first point.

With respect to the robustness of the method, Sects. 3.5, 3.6, 3.7, and 3.8 provide an estimation of the response of the method to distorted velocity readings, in both the inner and outer layers. It is shown that the accurate estimations of wall position and friction velocity are maintained even in worse situations of those expected in a careful experiment. A note of caution has to be made at this point; this robustness study has been made keeping the other parameters as a constant, in particular the position of the first point is the most critical one as stated in the previous paragraph. In case the measured profile does not reach $y^{+} \leq 10$, the possibility of the wall location should be disabled in the method. Another advantage is presented in an implicit way in the method description. It does not need the specification of the limits of the logarithmic layer. These limits span in the literature from the rather optimistic $30 \delta_{v} \leq y \leq 0.2 \delta$ to the quite conservative $200 \delta_{v} \leq y \leq 0.15 \delta$, and they present a high relevance in the estimation of the friction velocity by means of Clauser-like methods, specially at low Reynolds number. Therefore, the possibility of a method where a continuous description of the velocity profile is used will simplify these situations. The tendency followed by many authors (Table 1) has been to isolate the regions that may be affected by measurement problems (e.g., pressure gradient in the outer region or near-wall distorted measurements) when extrapolating inner or outer variables, respectively; this process was conducted by means of imposing somehow arbitrary limits to those regions. The robustness of the present method to noisy measurements permits the omission of these external inputs such as the limits of the logarithmic layer.

Concerning the Reynolds number, those that have been studied with DNS are on the same order of magnitude as the lowest Reynolds number available in the experimental data by Österlund (1999). The results obtained for the experimental validation at low Re suggest that the bump profile performs worse than the Musker profile in terms of detection of the wall stress $\tau_{w}$. However, when one considers even lower Reynolds number flows from the DNS dataset, it is clearly seen that the bump profile detects the correct shear stress with more accuracy. Concerning this point, one has to bear in mind that the accuracy of the measurements performed by Österlund (1999) is $\sim 1.5 \%$. This same error is obtained by the detection method; therefore, it is possible that the bump profile provides a better result (both for low and high Reynolds numbers) but the error present in the experimental method shows a bigger difference between the measured and the estimated shear stress.

Finally, a reminder is made again about the importance of an accurate comparison with published results. The method is sensitive to the velocity profile used and the definition of the error $E_{1}$ or $E_{2}$. A change in the definition of the error can account for up to $10 \%$ in the value of the wake parameter, П, Thus, when comparing with results from other papers, it is especially important to consider the same velocity profile and residual definition. This effect is just important for the descriptive parameters of the method, i.e., the wake component, $\Pi$, the von Kármán constant, $\kappa$, and the thickness of the boundary layer, $\delta$. Considering the values of the friction velocity and the wall position, the exact solution is known for the chosen comparison data; therefore, one can assess the accuracy of the method and evaluate which of the methods performs better. As mentioned in the previous sections, the method that provides the best results in terms on $u_{\tau}$ and $\Delta y$ is the bumpl, that is, 
the description of the velocity profile given by Eq. 4 with the bump given by Eq. 2 activated and the error definition given by Eq. 6 .

\section{Conclusion}

The proposed method allows the post-processing of the mean velocity profile in turbulent boundary layers. The main objective is the accurate estimation of the wall shear stress $\tau_{\text {wall }}$ and the wall position $\Delta y$. The inclusion of the von Kármán constant, $\kappa$, as a free parameter in the optimization enables an estimation of the friction velocity without making any previous assumption about the boundary conditions of the flow, which may have an effect on the von Kármán constant. Different methods have been tested concluding that the best results are provided by the bumpl method, which consider the overshoot over the logarithmic law in the buffer layer and provides a slightly larger weight to the points located close to the wall. Using this method and ensuring that the first measured point is located within the first $10 \delta_{v}$ from the wall, the accuracy of the method is $\Delta u_{\tau}= \pm 0.6 \%$ for the estimation of the friction velocity, $u_{\tau}$, and $\Delta y^{+}= \pm 0.3$ for the position of the wall, $\Delta y$ with an interval of confidence of $85 \%$ or $\Delta u_{\tau}= \pm 0.7 \%$ and $\Delta y^{+}= \pm 0.4$ with an interval of confidence of $95 \%$ . These results are, at least, as good as the most accurate way of determining the shear stress by experimental means, and they do not rely on any previously selected value for the von Kármán constant. In fact, it can be considered higher than any uncertainty found in experimental methods (e.g., $\Delta u_{\tau}=1.5 \%$ by Österlund (1999). The robustness of the method is also tested providing accurate results when including the fit pressure gradients, a poor data convergence, a reduced number of points, or distorted near-wall measurements. The only two hypotheses behind this method are the validity of the logarithmic law as a canonical description of the velocity profile (Eq. 4) and the experimental collapse of the data over the curve given by Eq. 3 presented by Nagib and Chauhan (2008). The method additionally determines the descriptive parameters of the boundary layer, $\Pi$ and $\delta$, which can be used as a diagnostic quantity to evaluate the validity of the performed experiment.

Acknowledgments The authors would like to acknowledge the financial support given by European Union FP7 Marie Curie MULTISOLVE project (Grant Agreement No. 317269).

Open Access This article is distributed under the terms of the Creative Commons Attribution License which permits any use, distribution, and reproduction in any medium, provided the original author(s) and the source are credited.

\section{References}

Alfredsson PH, Örlü R (2010) The diagnostic plot-a litmus test for wall bounded turbulence data. Eur J Mech B Fluids 29(6):403-406

Alfredsson PH, Örlü R, Schlatter P (2011) The viscous sublayer revisited-exploiting self-similarity to determine the wall position and friction velocity. Exp Fluids 51(1):271-280

Bailey SCC, Vallikivi M, Hultmark M, Smits a J (2014) Estimating the value of von Kármán constant in turbulent pipe flow. J Fluid Mech 749:79-98

Borrell G, Sillero Ja, Jiménez J (2013) A code for direct numerical simulation of turbulent boundary layers at high Reynolds numbers in BG/P supercomputers. Comput Fluids 80:37-43

Chauhan KA, Monkewitz PA, Nagib HM (2009) Criteria for assessing experiments in zero pressure gradient boundary layers. Fluid Dyn Res 41(2):021404

Chauhan KA, Nagib HM, Monkewitz PA (2007) On the composite logarithmic profile in zero pressure gradient turbulent boundary layers. In: 45th AIAA Aerospace Sciences Meeting and Exhibit, Reno, NV

Chew YT, Khoo BC, Li GL (1998) An investigation of wall effects on hot-wire measurements using a bent sublayer probe. Meas Sci Technol 9:67-85

Clauser FH (1954) Turbulent boundary laers in adverse pressure gradients. J Aeronaut Sci 21(2):91-108

Coles D (1956) The law of the wake in turbulent boundary layer. J Fluid Mech, 1:191-226

Coles D (1968) The young person's guide to the data. Computation of turbulent boundary layers, 1968 AFOSR-IFP Stanford Conference, vol. 2

Durst F, Kikura H, Lekakis I, Jovanovi J, Ye Q (1996) Wall shear stress determination from near-wall mean velocity data in turbulent pipe and channel flows. Exp Fluids 20:417-428

Fernholz HH, Janke G, Schober M, Wagner PM, Warnack D (1996) New developments and applications of skin-friction measuring techniques. Meas Sci Technol 7:1396-1409

Hutchins N, Choi K-S (2002) Accurate measurements of local skin friction coefficient using hot-wire anemometry. Prog Aerosp Sci $38: 421-446$

Jiménez J (2014) Direct numerical simulations of fully developed ZPG boundary layer flow $\left(R e_{\theta}=2780-6650\right)$. http://torroja.dmt. upm.es/turbdata/blayers/high_re. Accessed Oct 2014

Kendall A, Koochesfahani M (2008) A method for estimating wall friction in turbulent wall-bounded flows. Exp Fluids 44(5):773-780

Klewicki J (2007) Chapter 12.2: Measurement of wall shear stress. In: Tropea C, Yarin J, Foss A (eds) Handbook of experimental fluid mechanics. Springer, Berlin, pp 876-886

Marusic I, McKeon BJ, Monkewitz Pa, Nagib HM, Smits a J, Sreenivasan KR (2010) Wall-bounded turbulent flows at high Reynolds numbers: recent advances and key issues. Phys Fluids 22(6):1-24

Marusic I, Monty JP, Hultmark M, Smits AJ (2013) On the logarithmic region in wall turbulence. J Fluid Mech 716(1976):R3

McKeon BJ, Li J, Jiang W, Morrison JF, Smits a J (2004) Further observations on the mean velocity distribution in fully developed pipe flow. J Fluid Mech 501:135-147

Mehdi F, Johansson TG, White CM, Naughton JW (2013) On determining wall shear stress in spatially developing two-dimensional wall-bounded flows. Exp Fluids 55(1): 1656

Millikan C (1938) A critical discussion of turbulent flows in channels and circular tubes. In: Proceedings of the fifth international congress for applied mechanics, Harvard

Monkewitz PA, Chauhan KA, Nagib HM (2007) Self-consistent highreynolds-number asymptotics for zero-pressure-gradient turbulent boundary layers. Phys Fluids 19(11):1-12 
Musker A (1979) Explicit expression for the smooth wall velocity distribution in a turbulent boundary layer. AIAA J, 655-657

Nagib H, Christophoro C, Reudi J-d, Monkewitz P, Gravante S (2004) Can We Ever Rely on Results from Wall-Bounded Turbulent Flows without Direct Measurements of Wall Shear Stress? In Aerodynami Measurement Technology and Ground Testing Conference, number July. Portland, Oregon, pp 1-12

Nagib HM, Chauhan KA (2008) Variations of von Karman coefficient in canonical flows. Phys Fluids 20(10): 101518

Nagib HM, Chauhan Ka, Monkewitz P a (2007) Approach to an asymptotic state for zero pressure gradient turbulent boundary layers. Philos trans Ser A Math Phys Eng Sci 365:755-770

Nickels TB (2004) Inner scaling for wall-bounded flows subject to large pressure gradients. J Fluid Mech 521:217-239

Nocedal J, Wright S (2006) Numerical optimization, 2nd edn. Springer, London

Orlu R, Fransson JH, Henrik Alfredsson P (2010) On near wall measurements of wall bounded flows. The necessity of an accurate determination of the wall position. Prog Aerosp Sci 46(8):353-387

Österlund JM (1999) Experimental studies of zero pressure-gradient turbulent boundary-layer flow. PhD thesis, KTH, Royal Institute of Technology, Stockholm

Pope SB (2000) Turbulent flows. Cambridge University Press, Cambridge

Ryan MD, Ortiz-Dueñas C, Longmire EK (2011) Effects of simple wall-mounted cylinder arrangements on a turbulent boundary layer. AIAA J 49(10):2210-2220

Salari M, Tabar MM (2011) Accurate wall positioning of the hot-wire sensor using a high aspect-ratio rectangular nozzle. Flow Meas Instrum 22:138-143

Schlatter P (2014) Boundary layer DNS/LES Data. http://www.mech. kth.se/ pschlatt/DATA/\#DNS. Accessed Oct 2014

Schlatter P, Örlü R (2010) Assessment of direct numerical simulation data of turbulent boundary layers. J Fluid Mech 659:116-126

Schlatter P, Orlu R, Li Q, Brethouwer G, Fransson JHM, Johansson AV, Alfredsson PH, Henningson DS (2009) Turbulent boundary layers up to $R e_{\theta}=2500$ studied through simulation and experiment. Phys Fluids 21(5):51702

Schlichting H, Gersten K (2000) Boundary layer theory, 8th edn. Springer, London

Segalini A, Örlü R, Alfredsson PH (2013) Uncertainty analysis of the von Kármán constant. Exp Fluids 54(2):1460

Sillero Ja, Jiménez J, Moser RD (2013) One-point statistics for turbulent wall-bounded flows at Reynolds numbers up to $\delta^{+} \sim 2000$. Phys Fluids 25(10): 105102

Simens MP, Jiménez J, Hoyas S, Mizuno Y (2009) A high-resolution code for turbulent boundary layers. J Comput Phys 228(11):4218-4231

Tay CMJ, Khoo BC, Chew YT (2012) Determination of hot-wire position from a solid wall in an opaque channel. Meas Sci Technol 23:1-8

Vinuesa R (2013) Synergetic computational and experimental studies of wall-bounded turbulent flows and their two-dimensionality. $\mathrm{PhD}$ Thesis, Illinois Institute of Technology, Chicago

von Kármán T (1930) Mechanische Ahnlichkeit und Turbulenz. In: Proceedings of the 3rd international congress on applied mechanics. Stockholm, Sweden, pp 85-93

Watmuff J (1990) An experimental investigation of a low Reynolds number turbulent boundary layer subject to an adverse pressure gradient. In: Annual research briefs, 1989, vol 1, 37-49

Watmuff J (2014) Adverse pressure gradient agard database. http:// torroja.dmt.upm.es/turbdata/agard/chapter7/TBL12/. Accessed Jan 2015

Wei T, Schmidt R, McMurtry P (2005) Comment on the Clauser chart method for determining the friction velocity. Exp Fluids 38(5):695-699

Zagarola MV, Perry a E, Smits a J (1997) Log laws or power laws: the scaling in the overlap region. Phys Fluids 9(7):2094

Zanoun E-S, Durst F, Nagib H (2003) Evaluating the law of the wall in two-dimensional fully developed turbulent channel flows. Phys Fluids 15(10):3079 